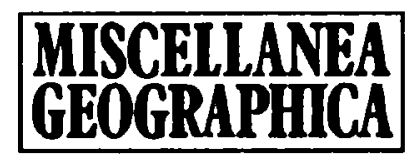

WARSZAWA 1994 Vol. 6

Jerzy Gilarowski

\title{
VÉGÉTATION ANCIENNE ET ACTUELLE EN AFRIQUE CENTRALE - CHANGEMENT ANTHROPIQUE DE L'ÉTENDUE DE LA FORÊT DENSE HUMIDE
}

D'après de nombreuses études géomorphologiques et palynologiques, au cours de deux derniers millénaires le climat de l'Afrique Centrale ne subissait pas de changements notables (Nicholson 1981, Roche 1991). Ses oscillations, de courte durée, n'avaient pas beaucoup d'influence sur l'extension de la forêt dense humide. Sa régression était due essentiellement à l'action exercée par l'homme. On est donc en droit de supposer que si les transformations anthropiques du milieu naturel n'avaient pas eu lieu, l'étendue de la forêt dense humide aurait été aujourd'hui sensiblement la même qu'il y a 2000 ans. Ajoutons à cela que la stabilisation du climat, après de profondes oscillations de l'Holocène inférieur et moyen, concorde dans le temps avec le début des immigrations des peuples agricoles bantous, venant du Nord vers l'Afrique Centrale. Jusque-là, elle était habitée par les Pygmées qui, se nourrissant surtout de venaison et de cueillette, ne transformaient guère le milieu naturel.

La comparaison de l'extension de la forêt dense humide d'il y a 2000 ans avec celle d'aujourd'hui aurait permis de mesurer l'étendue de la déforestation due à l'action de l'homme. Comment donc restituer ces anciennes limites? Les études palynologiques, pouvant donner les meilleurs preuves, sont trop rares pour pouvoir établir une carte détaillée de la végétation ancienne de l'Afrique Centrale. Il nous faut donc recourir à des données climatiques. Nous avons admis, comme nous l'avons fait pour l'Afrique de l'Ouest (Gilarowski 1992), que la forêt dense humide se développe dans les régions où la période de croissance des végétaux (PCV) est d'au moins 270 jours. En modifiant les données actuelles (augmentant de 15\% les valeurs des précipitations dans les terrains couverts aujourd'hui de savanes - puisque ces précipitations ont dû baisser avec la disparition de la forêt), nous avons donc tracé une isoligne de 260 jours de la PCV (la différence de 10 jours résulte de cette augmentation des précipitations) qui, d'après nos suppositions, correspond à l'étendue de la forêt dense humide d'il y a 2000 ans (fig. 1). Il y a lieu de remarquer que la ligne ainsi établie est 
sensiblement le même que la limite de la forêt dense humide tracée par $\mathbf{E}$. Roche et ses collaborateurs pour le Rwanda et le Burundi à l'aide de plusieurs analyses d'échantillons tirés de tourbières et marécages situés entre 1400 et $2100 \mathrm{~m}$ - donc utilisant des méthodes beaucoup plus précises; les différences ne dépassent pas, par endroits, $30-40 \mathrm{~km}$. La correspondance avec la carte de l'étendue de la forêt dense humide en Afrique Centrale, établie par E. Roche à petite échelle (Roche 1991), où ces limites sont alors très approximatives, est encore plus manifeste.

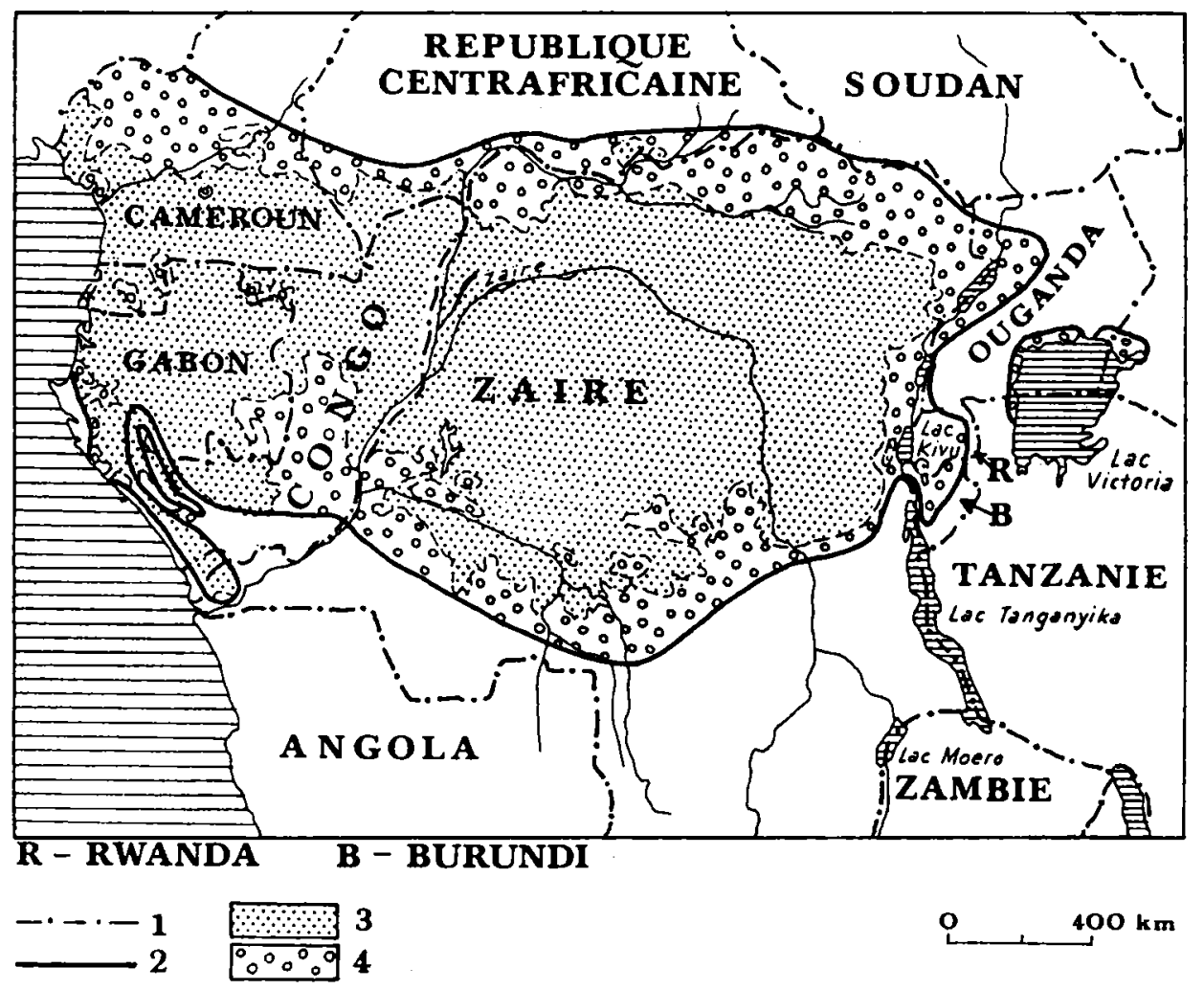

Fig. 1. L'étendue hypothétique de la forêt équatoriale d'avant 2 mille ans, en Afrique Centrale 1 - frontières d'États, 2 - isoligne de 260 jours de la PCV - limite de l'étendue de la forét équatoriale d'avant 2 mille ans, 3 - forêt equatoriale humide - étendue contemporaine, 4 aire déboisée pendant deux mille ans derniers

Pour la partie Sud-Ouest de la région, dans le Zaïre de l'Ouest, au Congo et au Gabon, le tracé a été modifié d'après les études sur les transformations de la végétation dans le Mayombé (Schwartz et allii 1990); en effet, à défaut de données météorologiques suffisantes et vu les différences climatiques importantes dans ces terrains du montagnes, la ligne de la PCV peut être difficilement considérée comme celle de l'étendue de la forêt. 
La comparaison entre l'extension de la forêt dense humide d'il y a 2 mille ans et son extension actuelle nous donne l'étendue de la déforestation provoquée par l'homme. Comme on le voit (fig. 1), la régression de la forêt n'était pas partout la même. La recul le plus important, de $300 \mathrm{~km}$ en moyenne, a eu lieu dans le Congo central et dans le Nord et le Nord-Est du Zaïre. Il était le moins sensible dans le Sud du Gabon et dans l'Ouest du Congo, où, dans le Mayombé, la forêt reprend par endroits sur la savane.

\section{BIBLIOGRAPHIE}

Gilarowski J., 1992, "Essai de reconstitution de l'étendue de la forêt dense humide en Afrique Occidentale d'avant la période agricole", Miscellanea Geographica 5, pp. 95-98, Warszawa.

Roche E., 1991, "Évolution des paléoenvironnements en Afrique centrale et orientale au Pléistocène et à l'Holocène. Influences climatiques et anthropiques", Bulletin de la Societé Géographique de Liège, 277 pp. 187-208, Liège.

$\mathrm{Schwartz} \mathrm{D}$. et allii, 1990, Paysages quaternaires de l'Afrique centrale atlantique, ORSTOM, Paris. 
